Originally published as:

Roma Schmitz, Susanne Jordan, Stephan Müters and Hannelore Neuhauser. Population-wide use of behavioural prevention and counselling programmes for lifestyle-related cardiovascular risk factors in Germany (2012) European Journal of Preventive Cardiology, August 19 (4), pp. 849-856.

DOI: $10.1177 / 1741826711410949$

This is an author manuscript.

The definitive version is available at: http://cpr.sagepub.com 


\title{
Population-wide use of behavioural prevention and counselling programmes for lifestyle-related cardiovascular risk factors in Germany
}

\author{
Roma Schmitz, Susanne Jordan, Stephan Müters and Hannelore Neuhauser
}

\begin{abstract}
Background: Behavioural prevention and counselling programmes for lifestyle-related cardiovascular risk factors are widely offered. However, their population-wide use is largely unexplored, particularly in high-risk persons with known CVD or diabetes.
\end{abstract}

Methods: Data were collected within GEDA 2009, a national health survey covering a representative sample of 21,262 adults in Germany. Standardised structured computer-assisted telephone interviews included self-reported physiciandiagnosed coronary heart disease, myocardial infarction, heart failure, stroke, diabetes as well as height, weight, usual diet, physical activity, and the use of programmes for weight reduction, healthy diet and improvement of fitness or mobility in the last twelve months.

Results: The use of prevention measures was lowest for weight reduction (men 2.1\%, women 3.5\%), somewhat higher for healthy diet (men 3.2\%, women 4.7\%), and highest for improvement of fitness or mobility (men 8.6\%, women 16.1\%). Among individuals with the respective risk factor, programme participation was still low: $5.5 \%$ in obese men (women $7.2 \%$ ) for weight reduction, $2.8 \%$ in men (women 3.7\%) who did not eat fruit or vegetables daily for healthy diet, and $7.9 \%$ in physically inactive men (women 15.7\%) for improvement of fitness or mobility. In the presence of known CVD or diabetes, participation increased inconsistently and only moderately.

Conclusion: Our results show low participation in behavioural prevention measures for lifestylerelated risk factors even in individuals with known CVD or diabetes. Further studies should investigate knowledge about potential programme benefit, availability and reimbursement in both patients and health care providers.

\section{Introduction}

Diseases of the cardiovascular system are of enormous public health relevance as they are the most common cause of premature invalidity and death. ${ }^{1}$ In 2008 , cardiovascular disease (CVD) accounted for more than $40 \%$ of all deaths in Germany. ${ }^{2}$ Being overweight or obese, having a diet low in fruit and vegetables and a sedentary lifestyle rank among the major risk factors for CVD.3,4 These are three of nine risk factors that account for more than $90 \%$ of acute myocardial infarctions according to the Interheart Study. ${ }^{5}$

To a great extent, development and progression of CVD is determined by individual behaviour and consumption patterns. In order to reduce cardiovascular risk, health organisations including the American Heart Association (AHA) and the World Health Organization (WHO) recommend maintaining a healthy diet and lifestyle for individuals in optimum cardiovascular health and reaching dietary and physical activity goals for persons at risk for CVD. ${ }^{6}$ In Germany, compulsory health insurance funds, which cover a large majority of the population, have had a legal mandate for primary prevention since 2000 and offer free (or reimbursement of) prevention courses irrespective of individual risk profile. ${ }^{7}$ Nutrition, physical activity and relaxation/coping with stress are counted among the central issues of prevention measures. ${ }^{8}$

While several studies evaluated counselling for diet and physical activity by physicians both for primary prevention of heart disease and in groups with highrisk co-morbidities such as diabetes and obesity,, 10 the population-wide use of behavioural prevention and counselling programmes for weight 
reduction, healthy diet and improvement of fitness and mobility is largely unexplored.

In particular, information on the use of these programmes by high-risk persons with already diagnosed cardiovascular diseases or diabetes is scarce. This study was aimed at investigating the use of behavioural prevention and counselling programmes for lifestyle-related cardiovascular risk factors in a large representative sample of the adult population in Germany.

\section{Methods}

The data of this study were collected within a national health interview survey, GEDA 2009, that assessed a large representative sample of the general non-institutionalised adult population in Germany aged 18 years or older (N1/421,262; 9148 men and 12,114 women).

Standardised structured computer-assisted telephone interviews were conducted by trained lay interviewers who had completed an extensive training programme, and were intermittently supervised and received refresher training.

To achieve a representative sample of the population, a modified random digit dialing sampling design for household selection ${ }^{11}$ was combined with a last-birthday method for selection of individual participants. In each household reached by landline telephone the interview was carried out with the person whose birthday was last. The cooperation rate at respondent level was $51.2 \%{ }^{12}$

GEDA covered various demographic and socioeconomic characteristics, physical health, lifestyle behaviours and health care utilisation. The participants were screened for the occurrence of cardiovascular diseases by asking them if they were ever diagnosed with diabetes mellitus, coronary heart disease, myocardial infarction, heart failure, and stroke using standardised questions of the following pattern: 'Did a physician ever diagnose ... ?'

For this study, participants were grouped into those who reported at least one of the above cardiovascular diseases or diabetes and those who did not. The question to evaluate the use of behavioural prevention and counselling programmes was: 'There are a number of prevention measures that are concerned with, e.g. nutrition, exercise, relaxation, and sport or fitness and that are offered by different providers.

The measures are partly paid by health insurances. Did you participate in such programmes, for instance, for weight reduction, a healthy diet, or improvement of fitness or mobility within the last twelve months?'.

Self-reported information about height and weight was used for calculating body mass index (BMI, in $\mathrm{kg} / \mathrm{m} 2$ ). Overweight was defined as BMI_25, and obesity as BMI_30. In accordance with the European Community Health Indicators Monitoring that uses the consumption of fruit and vegetables as an indicator for healthy diet, we classified diet as low in fruit or vegetables ('unhealthy diet') when less than daily use was reported. ${ }^{13}$

Physical activity (duration on the respective days per week) was assessed based on the International Physical Activity Questionnaire (IPAQ), a widely adopted instrument, and classified as insufficient in participants who reported less than three days per week with_30 min of moderate-intensity physical activity. ${ }^{14}$

Currently, the use of the IPAQ is tested for the EHIS, the European Health Indicator Survey. ${ }^{15}$ Statistical analyses were performed using SAS, Version 9.2 (SAS Institute Inc, Cary, NC). Prevalence and $95 \%$-confidence limits $(95 \% \mathrm{Cl})$ for lifestyle-related CVD risk factors and behavioural prevention programmes/ counselling were estimated using the survey commands in SAS. These commands account for survey design. Sampling weights were used to account for unequal sampling probabilities by household size and to reflect the distribution of the population with respect to age, sex, federal state and education. Any $95 \% \mathrm{Cl}$ that did not overlap were considered significantly different. All analyses were stratified by sex and also by age groups, except analysis of time trends. 


\section{Results}

In total, 21,262 persons (9148 men, 48.5\% and 12,114 women, 51.5\%) participated in GEDA 2009. For men, the population-wide use of behavioural prevention and counselling programmes within the last year was $2.1 \%(95 \% \mathrm{Cl} 1.8-2.5)$ for weight reduction, 3.2\% (2.8-3.7) for healthy diet and $8.6 \%$ (7.9-9.3) for improvement of fitness or mobility. Young men (18-44 years) had the lowest prevalence. For women, programme participation was higher than in men: $3.5 \%$ (3.1-3.9) for weight reduction, $4.7 \%(4.2-5.2)$ for healthy diet and $16.1 \%(15.3-16.9)$ for improvement of fitness or mobility.

Except for programmes for weight reduction, participation was again lowest in the youngest age group (18-44 years) (Table 1$)$.

The prevalence of being overweight was $43.4 \%$ in men and $29.3 \%$ in women, and the prevalence of obesity was $16 \%$ in both men and women. More than three-quarters of men $(76.1 \%)$ and more than half of women $(56.7 \%)$ reported less than daily use of fruit or vegetables. Additionally, $50.5 \%$ of men and $57.7 \%$ of women did not reach the physical activity level of _30 minutes on at least 3 days per week (Table 2).

Only $5.5 \%$ (3.9-7.2) of obese men and 7.2\% (5.7-8.7) of obese women participated in a weight reduction programme within the last year. While obesity increased with age in men and women, participation in a weight reduction programme among the obese did not. Programme participation was also low in men and women with low fruit and vegetable consumption $-2.8 \%(2.3-3.3)$ in men and $3.7 \%(3.1-4.2)$ in women - and somewhat higher in physically inactive men and women $-7.9 \%(7.0$ $8.9)$ in men and $15.7 \%(14.5-16.8)$ in women. Higher participation in women could be observed in almost all age groups (Table 2).

The lifetime prevalence of CVD/diabetes was $17.3 \%$ for men and $16.5 \%$ for women. For both sexes, the prevalence increased with age, but to a greater extent among men. In Table 3, men and women are grouped into those with CVD or diabetes and those without these diseases. The prevalence of cardiovascular risk factors and of the use of behavioural prevention and counselling programmes is shown for both groups. In general, women were more often users of programmes for weight reduction, healthy diet and improvement of fitness or mobility than men. However, if they hadCVD/diabetes, participation in specific programmes increased more in men than in women. This was most evident for a healthy diet programme $-7.6 \%(5.6-9.7)$ for men with CVD/diabetes vs. $1.9 \%(1.5-2.3)$ for men without CVD/diabetes. In women, the programme participation of physically inactive women with CVD/diabetes was even lower than that of physically inactive women without CVD/diabetes (Table 3).

\section{Trend over time (1998-2009)}

Figure 1 shows time trends in the use of programmes for weight reduction and healthy diet for men and women, separately. Data from GEDA 2009 were compared with data from the German National Health Interview and Examination Survey 1998 (BGS98), a representative sample of adults aged 1880 years. ${ }^{16}$ The population prevalence of the use of a programme for weight reduction increased by $163 \%$ in men and by $67 \%$ in women; for a healthy diet programme the increase was $167 \%$ in men and $81 \%$ in women. It is of note that participation in women remained higher than in men over the last decade (Figure 1).

\section{Discussion}

Based on data from the German National Health Interview Survey GEDA 2009 we report, for the first time, estimates of the population-wide use of behavioural prevention and counselling programmes for selected lifestyle-related cardiovascular risk factors.

We compare participation in men and women with and without known cardiovascular diseases or diabetes. Prevalence of participation in programmes was lowest for weight reduction (men $2.1 \%$, women 3.5\%), somewhat higher for healthy diet (men 3.2\%, women $4.7 \%$ ) and highest for improvement of fitness or mobility (men $8.6 \%$, women $16.1 \%$ ). As a proportion of individuals with the particular risk factor, programme participation was still low: for weight reduction in obese men it was $5.5 \%$ (women $7.2 \%$ ), for healthy diet in men who did not eat daily fruit or vegetables it was $2.8 \%$ (women $3.7 \%$ ) and for improvement of fitness or mobility in physically inactive men it was $7.9 \%$ 
(women 15.7\%). In the presence of known cardiovascular disease or diabetes participation increased inconsistently and only moderately.

Population-wide participation in programmes has not been reported yet. It is, however, of public health interest the proportion of the population that can be reached by such prevention measures. Moreover, in the presence of risk factors and particularly for those with known cardiovascular disease or diabetes, counseling is recommended by many relevant national and international organisations. In 2003, McTigue et al. ${ }^{17}$ reviewed the effectiveness of adult obesity screening and treatment and found that counselling promoted modest average weight loss. The US Preventive Services Task Force (USPCTF $)^{18,19}$ 'recommends that clinicians screen all adult patients for obesity and offer intensive counselling and behaviour interventions to promote sustained weight loss for obese adults'. Also in 2003, Pignone et al.20 performed an extensive systematic evidence review on behalf of the USPSTF aiming at evaluating whether counselling can improve dietary patterns. In this investigation, counselling was defined as a cooperative mode of interaction between the patient and primary care physician or related healthcare staff to assist patients in adopting behaviours associated with improved health outcomes.

The USPSTF ${ }^{18,19}$ concluded that 'the evidence is insufficient to recommend for or against routine behavioural counseling to promote a healthy diet in unselected patients in primary care settings'. However, for adults at high risk for CVD or diet-related chronic diseases intensive behavioural dietary counselling is recommended. With respect to physical activity, a review of controlled trials published since the 1996 USPSTF guidelines was performed by Eden et al.21 in 2002. The authors investigated whether adults counselled by primary care physicians improved or maintained physical activity behaviour and if so, what types of intervention were most effective. The USPSTF ${ }^{18,19}$ found insufficient evidence to determine whether counselling patients in primary care settings to promote physical activity leads to sustained increases in physical activity among adult patients, but recognises the effectiveness of physical activity to reduce chronic disease morbidity and mortality and acknowledges that many organisations recommend that healthcare providers counsel individuals about physical activity.

Overall, participation in behavioural counselling to promote weight reduction, a healthy diet and physical activity is low, even in the high-risk population. In fact, since cardiovascular disease and diabetes in this study is based on self-reported physician-diagnosed disease (and thereby likely underestimates the true proportions in the population), programme participation in highrisk groups is likely to be somewhat lower than reported. However, participation has increased considerably since 1998 for weight reduction (by a factor of 2.6 in men and 1.6 in women) and healthy diet (by a factor of 2.6 in men and 1.8 in women). This may be due to increased availability and new financial incentives created in 2000 by compulsory health insurances, which cover almost $90 \%$ of the German population. These incentives include reimbursement of primary prevention measures in the area of nutrition, physical activity and coping with stress and are not related to disease or risk status. ${ }^{8}$

The BGS98 on the other hand refers to a time period with mostly reduced availability of programmes. The increase was greater in men than in women, but men still did not catch up on absolute participation despite a higher prevalence of overweight/ obesity and unhealthy diet defined as a consumption of fruit or vegetables less than daily.

The strengths of this study are the large, nationally representative and unselected sample covering a wide age range and the standardised questions allowing for a comparison with the national health survey from 1998. However, GEDA 2009 and BGS98 are not fully comparable since one used computer-assisted telephone interviews and the other questionnaires and physician interviews and sampling frames differed. A limitation of the study is the lack of more detailed information on the content and structure of the programmes. The wording of the question in German, however, clearly refers to a comprehensive activity and not merely a one-time discussion of the topic in primary care.

Another potential limitation of this study is selection bias resulting from non-response and from recruitment via fixed-line telephones only. According to the German National Statistical Office, the proportion of cell phone-only households in Germany more than doubled between 2003 and 2008. However, it still accounts for only $9 \%$ of all households in Germany and is one of the lowest in Europe. ${ }^{22}$ An additional limitation is self-reporting of programme participation, health behaviours and diagnoses that may be influenced by recall bias, by social desirability bias (in particular for health behaviours) and by limited sensitivity and specificity of self- 
reported diagnoses. A comprehensive study on the validity and reliability of a wide range of topics from the computer-assisted telephone interview surveys conducted within the Behavioural Risk Factor Surveillance System (BRFSS) in the US concluded that the core BRFSS questions were at least moderately reliable and valid, and many were highly reliable and valid.

Intense leisure-time physical activity and fruit and vegetable consumption were among the items with moderate reliability and validity while body mass index had high reliability and validity. ${ }^{23}$ However, these potential biases are an unlikely explanation for the full extent of our findings.

Overall, our results show low participation in behavioural prevention and counselling programmes for lifestyle-related risk factors even in men and women with known cardiovascular disease or diabetes. Further studies should investigate knowledge in both patients and physicians and other health care providers about the potential benefits of programmes as well as on their availability and on the reimbursement option.

\section{Funding}

This research received no specific grant from any funding agency in the public, commercial or not-forprofit sectors.

\section{Conflicts of interest}

None declared. 


\section{References}

1. Mathers CD, Boerma T and Ma Fat D. Global and regional causes of death. Br Med Bull 2009; 92: 7-32.

2. Statistisches Bundesamt. Gesundheit - Todesursachen in Deutschland. Fachserie 12, Reihe 4. Wiesbaden, 2010.

3. Graham I, Atar D, Borch-Johnsen K, Boysen G, Burell G, Cifkova R, et al. European guidelines on cardiovascular disease prevention in clinical practice: executive summary. Fourth Joint Task Force of the European Society of Cardiology and other societies on cardiovascular disease prevention in clinical practice (constituted by representatives of nine societies and by invited experts). Eur J Cardiovasc Prev Rehabil 2007; 14(Suppl 2): E1-E40.

4. Lloyd-Jones DM, Hong Y, Labarthe D, Mozaffarian D, Appel LJ, Van Horn L, et al. Defining and setting national goals for cardiovascular health promotion and disease reduction: the American Heart Association's strategic Impact Goal through 2020 and beyond. Circulation 2010; 121: 586-613.

5. Yusuf S, Hawken S, Ounpuu S, Dans T, Avezum A, Lanas F, et al. Effect of potentially modifiable risk factors associated with myocardial infarction in 52 countries (the INTERHEART study): case-control study. Lancet 2004; 364: 937-952.

6. Labarthe DR. Epidemiology and prevention of cardiovascular diseases: a global challenge, 2nd ed. Sudberry: Jones and Bartlett Publishers, 2010.

7. Arbeitsgemeinschaft der Spitzenverbände der Krankenkassen. Task Force of the Central Organisation Health Insurance. Guideline Prevention. Areas of action and criteria of the Central Organisation to serve $\S \S 20$ und 20a SGB V of 21 June 2000. Bonn. Frankfurt am Main: KomPart Publishing, 2010.

8. Medizinischer Dienst des Spitzenverbandes Bund der Krankenkassen e. V. (MDS), GKV-Spitzenverband. Medical Service of the Central Organisation of the Health Insurers' Alliance, Central Organisation Compulsury Health Insurance. Prevention Report 2010. Benefits of the Compulsury Health Insurance: Primary Prevention and Workplace Health Promotion. Essen, 2010. Berichtsjahr 2009. Essen: MDS, GKVSpitzenverband, 2010.

9. Centers for Disease Control and Prevention. Missed opportunities in preventive counseling for cardiovascular disease - United States, 1995. MMWR Morb Mortal Wkly Rep 1998; 47: 91-95.

10. Stafford RS, Farhat JH, Misra B and Schoenfeld DA. National patterns of physician activities related to obesity management. Arch Fam Med 2000; 9: 631-638.

11. Gabler S and Ha" der S. Generating Telephone Samples with TelSuSa. ZUMA-Nachrichten 1999; 44: 138143.

12. Robert Koch-Institut. Daten und Fakten: Ergebnisse der Studie 'Gesundheit in Deutschland aktuell 2009'.

Robert Koch Institute. Data and facts: Results of the study "Health in Germany 2009". Beiträge zur

Gesundheitsberichterstattung des Bundes. Berlin: Robert Koch-Institut, 2010.

13. Kilpeläinen $\mathrm{K}$ and Aromaa A (the ECHIM project). European health indicators: development and initial implementation. Final report of the ECHIM project. Helsinki: Helsinki University Press, 2008.

14. Craig CL, Marshall AL, Sjostrom M, Bauman AE, Booth ML, Ainsworth BE, et al. International physical activity questionnaire: 12-country reliability and validity.

Med Sci Sports Exerc 2003; 35: 1381-1395.

15. http://www.healthindicators.eu/healthindicators/object_document/05987n29063.html (accessed April 2011).

16. Bellach BM, Knopf H and Thefeld W. The Federal Health Survey 1997/98. Gesundheitswesen 1998;

60(Suppl 2): S59-S68.

17. McTigue KM, Harris R, Hemphill B, Lux L, Sutton S, Bunton AJ, et al. Screening and interventions for obesity in adults: summary of the evidence for the US Preventive Services Task Force. Ann Intern Med 2003; 139: 933949.

18. Agency for Healthcare Research and Quality. Guide to Clinical Preventive Services, 2010-2011.

Recommendations of the US Preventive Services Task Force. http://www.ahrq.gov/clinic/pocketgd.htm (accessed April 2010).

19. US Preventive Services Task Force (USPSTF). USPSTF Recommendations.

http://www.uspreventiveservicestask force.org/uspstopics.htm (accessed April 2010).

20. Pignone MP, Ammerman A, Fernandez L, Orleans CT, Pender N, Woolf S, et al. Counseling to promote a healthy diet in adults: a summary of the evidence for the US Preventive Services Task Force. Am J Prev Med 2003; 24: 75-92.

21. Eden KB, Orleans CT, Mulrow CD, Pender NJ and Teutsch SM. Does counseling by clinicians improve physical activity? A summary of the evidence for the US Preventive Services Task Force. Ann Intern Med 2002; 137: 208-215.

22. http://www.destatis.de/jetspeed/portal/cms/Sites/destatis/ Internet/DE/Presse/pm/2009/05/PD09_184_632.psml (accessed May 2011).

23. Nelson DE, Holtzman D, Bolen J, Stanwyck CA and Mack KA. Reliability and validity of measures from the Behavioral Risk Factor Surveillance System (BRFSS). Soz Praventivmed 2001; 46(Suppl 1): S3-S42. 


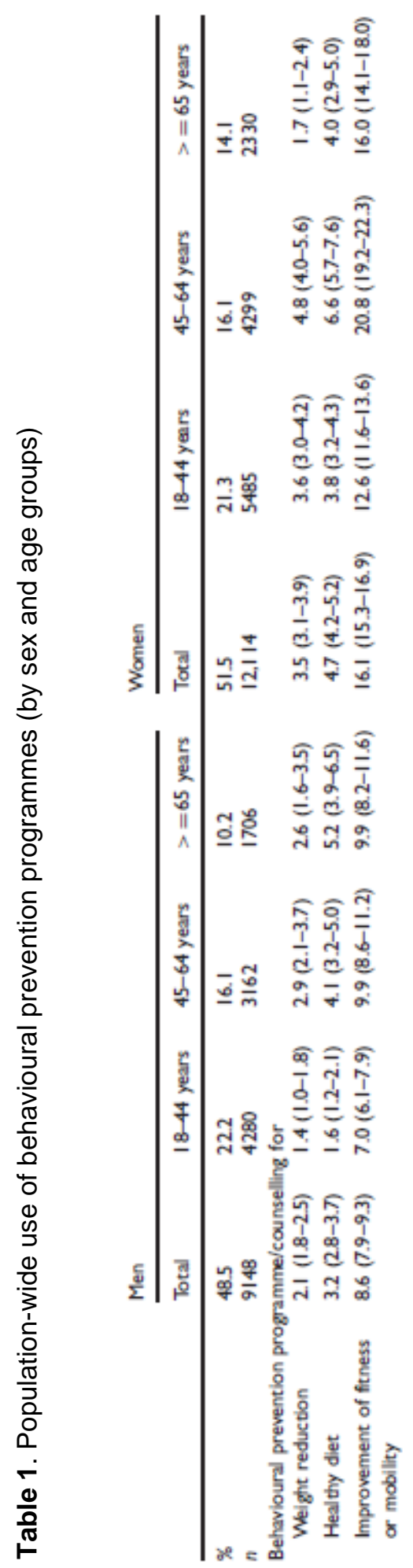




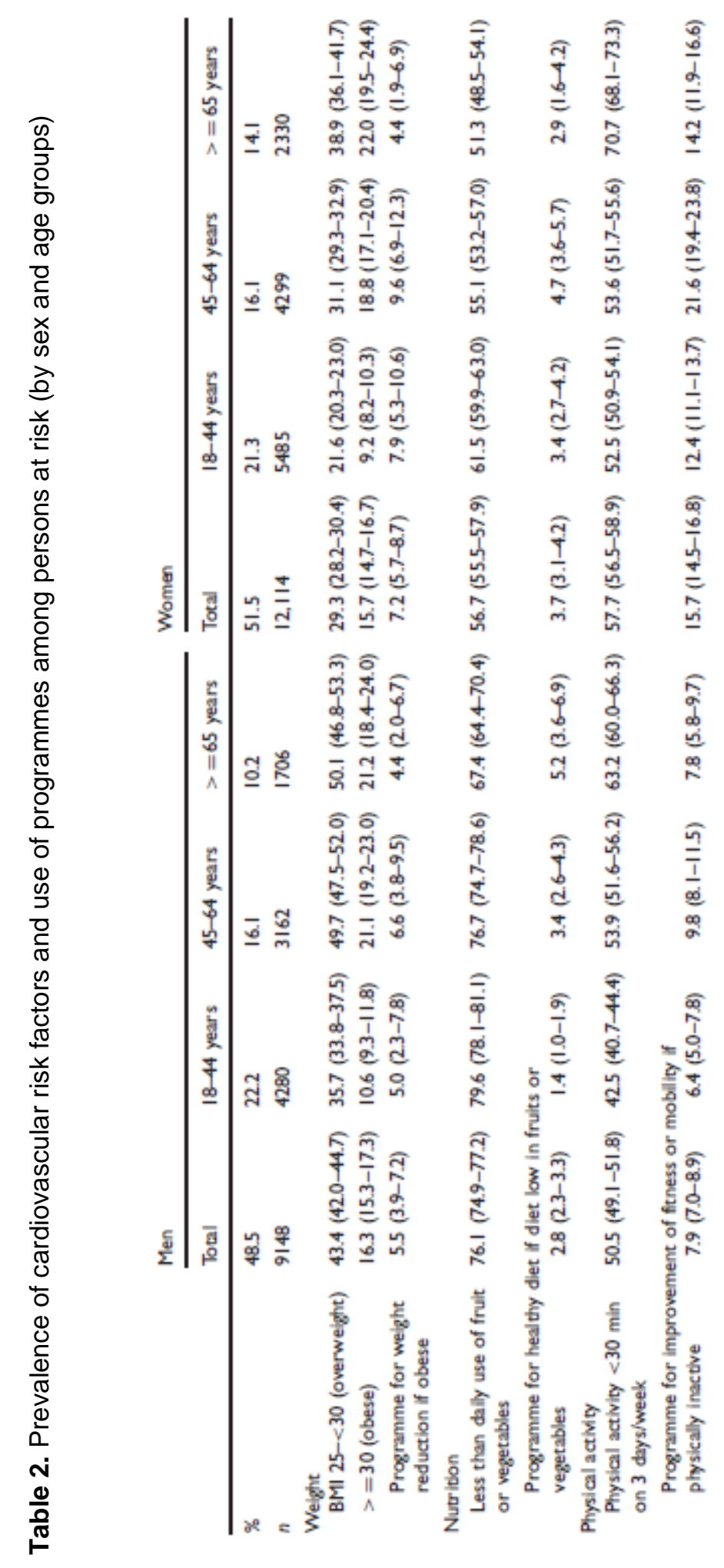




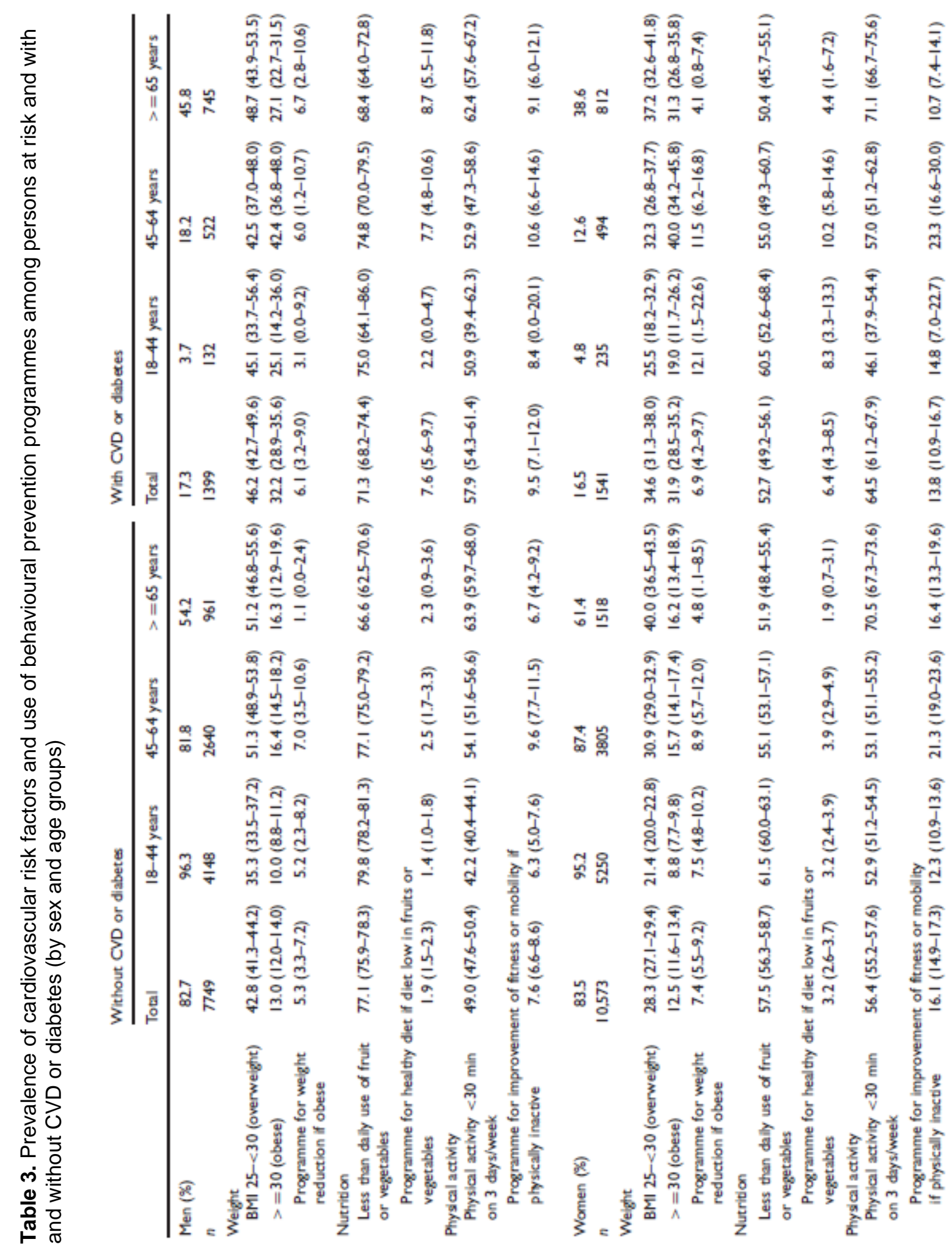


Figure 1. Time trend in use of behavioural prevention programmes/counselling (population prevalence in \%).

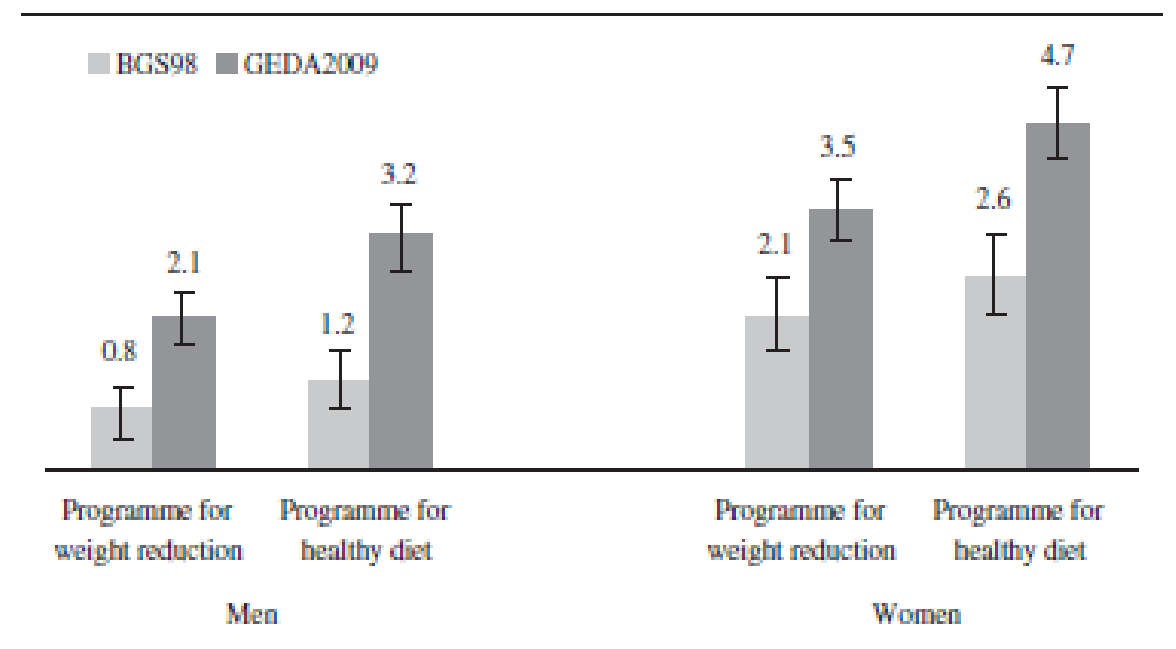

